\title{
Cellular electrodynamic activity
}

\author{
Michal Cifra ${ }^{1, a}$, Daniel Havelka ${ }^{1}$ and Ondřej Kučera ${ }^{1}$
}

${ }^{1}$ Institute of Photonics and Electronics, The Czech Academy of Sciences, 18200 Prague, Czech Republic

\begin{abstract}
Electromagnetic activity of cells $(>100 \mathrm{kHz})$ beyond the conventional frequency region of electrophysiology is poorly explored, yet it offers opportunities for new diagnostic and therapeutic methods in bioelectronic medicine and may provide insights into new general mechanism in cellular signalling. Here, we describe electromagnetic properties of microtubules, protein nanostructures omnipresent in biological systems, and how they are predicted to be involved in cellular electromagnetic activity $(10 \mathrm{MHz}-10 \mathrm{GHz})$.

Authors were supported from institutional funding of the Institute of Photonics and Electronics, The Czech Academy of Sciences and by the Czech Science Foundation, grant no. 15-17102S.
\end{abstract}

${ }^{a}$ Corresponding author: cifra@ufe.cz 